\title{
Connectedness of approximate weak efficient sets for the
}

\section{set-vector equilibrium problem}

\author{
Yunxuan Xiong \\ ${ }^{1}$ Nanchang Institute of Science and Technology, Nanchang 330108, Jiangxi \\ xyx38@126.com
}

Keywords: Vector equilibrium problem; Approximate weak efficient; Connectedness

\begin{abstract}
In this paper, a scalarization result of approximate weak efficient solution for a set-vector equilibrium problem (SVEP) is given. With this scalarization result, the connectedness of $\varepsilon$-weak efficient sets for the SVEP is proved under some suitable conditions in real Hausdorff topological vector spaces. The main results presented in this paper improve and generalize some known results in the literature $[6,7,10]$.
\end{abstract}

\section{Introduction}

Vector equilibrium problems were first introduced by Blum and Oettli, It is well known that several problems such as vector optimization problems, fixed point problems, vector variational problems, and Nash economic equilibrium problems can be studied as particular cases of vector equilibrium problems.

Vector equilibrium problems research mainly includes the existence of the solution, the stability of the solution sets, sensitivity analysis, topological properties of the set of solutions etc. The solution of the connectedness is an important aspect of topological properties, because it provides the possibility of continuously moving from one solution to any other solution. Recently, Lee et al. [2],Cheng [3] have studied the connectedness of weak efficient solutions set for vector variational inequalities in finite dimensional Euclidean space. Gong [4-6] has studied the connectedness of the various solutions set for VEPs in infinite dimension space. Chen et al. [7] studied the connectedness and the compactness of the weak efficient solutions set for set-valued VEPs and the set-valued vector Hartman-Stampacchia variational inequalities in normed linear space. Gong and Yao [8] have studied the connectedness of the set of efficient solutions for generalized systems. Zhong et al. [9] have studied the connectedness and path-connectedness of solutions set for symmetric VEPs. Chen et al. [10] studied the connectedness of approximate solutions set for vector equilibrium problems in Hausdorff topological vector spaces .and Chen Bin et al have studied the connected of the single value of vector equilibrium problems.

This paper inspired by the paper[6,7,10],studies the connectedness and compactness of approximate weak efficient sets for the set-valued vector equilibrium problem under different conditions ,thus promoting the conclusion of paper $[6,7,10]$ 


\section{Preliminaries}

Throughout this paper, let $X, Y$ be two real Hausdorff topological vector spaces, $C$ be a closed convex pointed cone in $Y$ and int $C \neq \varnothing, A$ be a nonempty subset of $X$,Let $F: A \times A \rightarrow 2^{Y}$ be a set-valued mapping,

$C^{*}=\left\{f \in Y^{*}: f(y) \geq 0, \forall y \in C\right\}$ be the dual cone of C. Denote the quasi-interior of $C^{*}$ by $C^{\#}$.that is $C^{\#}=\left\{f \in Y^{*}: f(y) \geq 0, \forall y \in C \backslash\{0\}\right\}$.let $e$ be a fixed point in int $C$ and we set $C^{\prime}=\left\{f \in C^{*} \backslash\{0\}: f(e)=1\right\}, C^{\prime \prime}=\left\{f \in C^{\#}: f(e)=1\right\}$.

Definition 2.1 If $\operatorname{int} C \neq \varnothing$, the vector $x \in A$ satisfying $F(x, y) \cap(-\operatorname{int} C-\varepsilon e)=\varnothing$ for all $y \in A$,is called $\varepsilon$-weak efficient solution to the SVEP. Denote by $V_{\varepsilon-W}(A, F)$ the set of all $\varepsilon$-weak efficient solutions to the SVEP.

Definition 2.2 The vector $x \in A$ satisfying $F(x, y) \cap(-C \backslash\{0\}-\varepsilon e)=\varnothing$ for all $y \in A$ is called $\varepsilon$-efficient solution to the SVEP. Denote by $V_{\varepsilon}(A, F)$ the set of all $\varepsilon$-efficient solutions to the SVEP.

Definition 2.3 Let $f \in C^{*} \backslash\{0\}$, vector $x \in A$ satisfying $f(F(x, y)) \geq \varepsilon$, for all $y \in A$ is called $\varepsilon-f$ efficient solution to the SVEP and Denote by $V_{\varepsilon-f}(A, F)$ the set of all $\varepsilon-f$ efficient solutions to the SVEP.

Definition 2.4 let $X$ be a Hausdorff topological vector spaces, and $E$ be a nonempty convex subset of $X$,the set-valued mapping $G: E \rightarrow 2^{X}$ is called KKM mapping if for any finite set $\left\{x_{1}, x_{2}, \cdots x_{n}\right\} \subset E$, then the relation $\operatorname{co}\left\{x_{1}, x_{2}, \cdots x_{n}\right\} \subset \bigcup_{i=1}^{n} G\left(x_{i}\right)$ hold. where $\operatorname{co}\left\{x_{1}, x_{2}, \cdots x_{n}\right\}$ denoted the convex hull of $\left\{x_{1}, x_{2}, \cdots x_{n}\right\}$.

Lemma2.1 let $X, Y, Z$ be two real Hausdorff topological vector spaces, $G: Y \rightarrow Z$ is a single-valued mapping, $F: X \rightarrow 2^{Y}$ is a set-valued mapping, if $F$ is upper semicontinuous in $X, G$ is lower semi-continuous in $Y$, then $G \circ F: X \rightarrow 2^{Z}$ is lower semi-continuous in $X$.

Lemma2.2 let $A \subset X$ be a nonempty connected set ,let $F: A \rightarrow 2^{Y}$ be an upper 
semi-continuous in $A$, and for all $x \in A, F(x)$ be a connected value, then $\bigcup_{x \in X} F(x)$ is connected.

Lemma2.3 ${ }^{[13]}$ let $X, Y$ be two Hausdorff topological vector spaces, set-valued mapping $F: X \rightarrow 2^{Y}$ be an lower semi-continuous at $x_{0} \in X$ if and only if for any net $\left\{x_{\alpha}\right\}, x_{\alpha} \rightarrow x_{0}$,and for any $y \in F\left(x_{0}\right)$,there exists a net $\left\{y_{\alpha}\right\}, y_{\alpha} \in F\left(x_{\alpha}\right)$ such that $y_{\alpha} \rightarrow y$.

Lemma2.4 (FKKM Theorem) let $E$ be a nonempty subset of $X$,if $G: E \rightarrow 2^{X}$ is KKM mapping, $G(x)$ is closed set for all $x \in E$, and there is at least exist $x_{0} \in E$ with $G\left(x_{0}\right)$ is a compact set then $\underset{x \in K}{\cap} G(x) \neq \varnothing$.

Lemma2.5 Suppose int $C \neq \varnothing$, and $F(x, A)+C$ is a convex set for each $x \in A$.then $V_{\varepsilon-W}(A, F)=\bigcup_{f \in C^{\prime}} V_{\varepsilon-f}(A, F)$

Proof. The proof is the similar with Theorem 3.1.of the paper[7].

Lemma2.6 let $X, Y$ be two Hausdorff topological vector spaces, $C$ is a closed convex pointed cone in $Y$ and int $C \neq \varnothing, A$ is a nonempty compactness subset of $X, F: A \times A \rightarrow 2^{Y}$ is a set-valued mapping, $f \in C^{\prime}$. Assume that the following conditions are satisfied.

(i) $x \in A, F(x, x) \subset C-\varepsilon e$, for all $x \in A$;

(ii) For each fixed $y \in A, F(\cdot, y)$ is lower semi-continuous on $A$;

(iii) $F(x, y)$ is $C$ quasi-convex-like with respect to the second variable;

Then $V_{\varepsilon-f}(A, F) \neq \varnothing$.

Proof. It can be easily proved with the Lemma2.4, thus omitted.

\section{Connectedness of the solutions set}

Theorem 3.1 let $X, Y$ be two Hausdorff topological vector spaces , $C$ is a closed convex pointed cone in $Y$ andint $C \neq \varnothing, A$ is a nonempty compactness subset of 
$X, F: A \times A \rightarrow 2^{Y}$ is a set-valued mapping, $f \in C^{\prime}$. Assume that the following conditions are satisfied.

(i) $F(x, x) \subset C-\varepsilon e$, for all $x \in A$;

(ii) For each fixed $y \in A, F(\bullet, y)$ is lower semi-continuous on $A$;

(iii) $F(x, y)$ is $C$ quasi-convex-like with respect to the first and second variable; and quasi-convex-like with respect to the second variable;

(iv) $\{F(x, y): x, y \in E\}$ is a bounded set of $Y$.

Then $V_{\varepsilon-W}(A, F)$ is a connectedness set .

Proof. By Lemma2.5 we know $V_{\varepsilon-f}(A, F) \neq \varnothing$.

First we proof $V_{\varepsilon-f}(A, F)$ is a connected set. Let $x_{1}, x_{2} \in V_{\varepsilon-W}(A, F)$, then we have $x_{1}, x_{2} \in A, f\left(F\left(x_{i}, y\right)\right) \geq-\varepsilon, \forall y \in A, i=1,2$.Since $F(x, y)$ is $C$ quasi-convex-like with respect to the first variable, then we have

$F\left(t x_{1}+(1-t) x_{2}, y\right) \subset F\left(x_{1}, y\right)+C$ or $F\left(t x_{1}+(1-t) x_{2}, y\right) \subset F\left(x_{2}, y\right)+C$ is true.

Now we suppose $F\left(t x_{1}+(1-t) x_{2}, y\right) \subset F\left(x_{1}, y\right)+C$ is true. Then let $z \in F\left(t x_{1}+(1-t) x_{2}, y\right)$, we have $z \in F\left(x_{1}, y\right)+C$.Then there exist $z^{\prime} \in F\left(x_{1}, y\right)$ and $c^{\prime} \in C$, such that $\mathrm{z}=\mathrm{z}^{\prime}+c^{\prime}$ Since $x_{1}, x_{2} \in V_{\varepsilon-W}(A, F)$, we obtain $f\left(z^{\prime}\right) \geq-\varepsilon$.

Because $c^{\prime} \in C$, then $f\left(c^{\prime}\right) \geq 0$,Hence $f(z)=f\left(z^{\prime}+c^{\prime}\right)=f\left(z^{\prime}\right)+f\left(c^{\prime}\right) \geq-\varepsilon$.In other words $f\left(F\left(t x_{1}+(1-t) x_{2}, y, \lambda\right)\right) \geq-\varepsilon$. Thus we obtain $t x_{1}+(1-t) x_{2} \in V_{\varepsilon-W}(A, F)$. So $V_{\varepsilon-f}(A, F)$ is a convex set, thus $V_{\varepsilon-f}(A, F)$ is a connected set.

Next we proof $V_{\varepsilon-W}(A, F)$ is a connected set.

Define a set-valued Mapping $H: C^{\prime} \rightarrow 2^{E}$ by $H(f)=V_{\varepsilon-f}(A, F)$.By lemma2.5, we show that $H$ is upper semi-continuous on $C^{\prime}$.

Since $A$ is a compact set, then we will proof $H$ is Closed.

Let $\left\{\left(f_{\alpha}, x_{\alpha}\right)\right\} \subset \operatorname{Graph}(H)$ and $\left(f_{\alpha}, x_{\alpha}\right) \rightarrow\left(f_{0}, x_{0}\right)$, where $\left\{f_{\alpha}\right\}$ converges to $f_{0}$ with respect to the strong topology $\beta\left(Y^{*}, Y\right)$. Then we have $x_{\alpha} \in H\left(f_{\alpha}\right)=V_{\varepsilon-f_{\alpha}}(A, F)$. 
It is means that $f_{\alpha}\left(F\left(x_{\alpha}, y\right)\right) \geq-\varepsilon, \forall y \in A$.

Since $x_{\alpha} \rightarrow x_{0}, A$ is a compact set, then $x_{0} \in A$. By assumption for each $y \in E$, $F(\bullet, y)$ is lower semi-continuous on $A$, let $y \in E, z_{0} \in F\left(x_{0}, y\right)$, there exist $z_{\alpha} \in F\left(x_{\alpha}, y\right)$ such that $z_{\alpha} \rightarrow z_{0}$. Since $f_{\alpha}\left(F\left(x_{\alpha}, y\right)\right) \geq-\varepsilon$, we have $f_{\alpha}\left(z_{\alpha}\right) \geq-\varepsilon$.

Let $Q=\{F(x, y): x, y \in E\}$.since $p_{Q}(f)=\sup _{x \in Q}|f(x)|$ is a continuous semi-norm in $\beta\left(Y^{*}, Y\right)$, For any $\varepsilon>0, U_{\delta}=\left\{f \in Y^{*}: p_{Q}(f)<\delta\right\}$ is a neighborhood of 0 with respect with to $\beta\left(Y^{*}, Y\right)$.And so for $\delta>0, f_{\alpha} \rightarrow f_{0}$, there exist $\alpha_{0}^{1}$, when $\alpha \geq \alpha_{0}^{1}$ we have $\left|f_{\alpha}\left(z_{\alpha}\right)-f_{0}\left(z_{\alpha}\right)\right|=\left|\left(f_{\alpha}-f_{0}\right)\left(z_{\alpha}\right)\right|<\delta$.Since $z_{\alpha} \rightarrow z_{0}$, there exist $\alpha_{0}^{2}$,when $\alpha \geq \alpha_{0}^{2}$ we have $\left|f_{0}\left(z_{\alpha}\right)-f_{0}\left(z_{0}\right)\right|<\delta$.Take $\alpha_{0}$ satisfying $\alpha \geq \alpha_{0}^{1}$ and $\alpha \geq \alpha_{0}^{2}$, when $\alpha \geq \alpha_{0}$ we can get $\left|f_{\alpha}\left(z_{\alpha}\right)-f_{0}\left(z_{0}\right)\right| \leq\left|f_{\alpha}\left(z_{\alpha}\right)-f_{0}\left(z_{\alpha}\right)\right|+\left|f_{0}\left(z_{\alpha}\right)-f_{0}\left(z_{0}\right)\right|<2 \delta$. Then $f_{\alpha}\left(z_{\alpha}\right) \rightarrow f_{0}\left(z_{0}\right)$.

Because $f_{\alpha}\left(z_{\alpha}\right) \geq-\varepsilon$, then we have $f_{0}\left(z_{0}\right) \geq-\varepsilon$.thus $f_{0}\left(F\left(x_{0}, y, \lambda\right)\right) \geq-\varepsilon$.By the arbitrary of $y$, we know $x_{0} \in H\left(f_{0}\right)$, so $H$ is a closed mapping. Thus $H$ is a upper semi-continuous .by lemma2.2, we know $V_{\varepsilon-W}(A, F)$ is a connected set.

\section{Remark:}

(1) When $\varepsilon=0$, the $\varepsilon$-weak efficient solution to the SVEP is reduce to weak efficient solution to the SVEP, which have been studied in [7].

(2) When set-valued mapping $F: A \times A \rightarrow 2^{Y}$ reduce to single mapping $f: A \times A \rightarrow$ $Y$,which have been studied in [10].

\section{References}

[1] Blum, Eugen, and Werner Oettli. From optimization and variational inequalities to equilibrium problems. Mathematics Student-India .63 (1994): 123-145.

[2] Lee, Gue Myung, Byung Soo Lee, and Nguyen Dong Yen. Vector variational inequality as a tool for studying vector optimization problems.Nonlinear Analysis: Theory, Methods \& Applications .34 (1998): 745-765.

[3] Cheng, Yonghong. On the connectedness of the solution set for the weak vector variational inequality. Journal of Mathematical Analysis and Applications. 260 (2001): 1-5. 
[4] Gong X H. Efficiency and Henig efficiency for vector equilibrium problems. Journal of Optimization Theory and Applications. 108(2001) 139-154.

[5] Gong X H, Fu W T, Liu W. Super efficiency for a vector equilibrium in locally convex topological vector spaces. Vector variational inequalities and vector equilibria. (2000). 233 - 252.

[6] Gong, X. H. Connectedness of the solution sets and scalarization for vector equilibrium problems. Journal of Optimization Theory and Applications. 133 (2007): 151-161.

[7] Chen B,Gong X H,Yuan S M. Connectedness and compactness of weak efficient solutions for set-valued vector equilibrium problems. Journal of Inequalities and Applications. 15. (2007): 1-15.

[8] Gong X H,Yao J C. Connectedness of the set of efficient solutions for generalized systems. Journal of Optimization Theory and Applications.138 (2007): 189-196.

[9] Zhong R,Huang N,Wong M M. Connectedness and path-connectedness of solution sets to symmetric vector equilibrium problems. Taiwanese Journal of Mathematics.13 (2009). 821-836.

[10]Chen B,Liu Q,Liu Z,et al. Connectedness of approximate solutions set for vector equilibrium problems in Hausdorff topological vector spaces. Fixed Point Theory and Applications. 2011 (2011). 1-11.

[11] Arrow K J,Debreu G. "Existence of an equilibrium for a competitive economy". Econometrica: Journal of the Econometric Society. 22 (1954). 265-290.

[12] Warburton A R. Quasiconcave vector maximization: connectedness of the sets of Pareto-optimal and weak Pareto-optimal alternatives. Journal of optimization theory and applications. 40(1983) 537-557.

[13] Ferro F. Optimization and stability results through cone lower semicontinuity. Set-Valued Analysis. 5(1997). 365-375.

[14]Muselli E. Upper and lower semicontinuity for set-valued mappings involving constraints. Journal of Optimization Theory and Applications. 106(2000). 527-550. 